# EFECTO DEL CONTENIDO DE HUMEDAD Y TEMPERATURA SOBRE LA DIFUSIVIDAD TÉRMICA EN GRANOS ANDINO 

Nils L. Huamán Castilla*a, Gladys Yupanquii ${ }^{\mathrm{b}}$, Erik Allcca ${ }^{\mathrm{a}}$, Giovanna Allcca ${ }^{\mathrm{c}}$


#### Abstract

RESUMEN Este estudio muestra un método sencillo para evaluar la difusividad térmica $(\alpha)$ en granos andinos de quinua (Chenopodium quinoa Willd) y cañihua (Chenopodium pallidicaule Aellen). Además, se estudió el efecto de la temperatura de 25 a $35^{\circ} \mathrm{C}$ y humedad en base húmeda de 10 a $20 \%$ sobre el comportamiento de esta propiedad. El método empleado para determinar la difusividad fue el propuesto por Gupta, el cual hace uso de las curvas de penetración de calor en un equipo especialmente armado para este fin. Los resultados obtenidos indican que los valores varían entre 7,5 y $8,9 \times 10^{-8} \mathrm{~m}^{2} . \mathrm{s}^{-1}$. La evaluación de los datos indica que existe una fuerte influencia del tipo de grano, la humedad y la temperatura en esta propiedad térmica. Adicionalmente, se usó la metodológica de superficie de respuesta para encontrar un modelo empírico para representar la difusividad térmica en función del contenido de humedad y temperatura.


Palabras clave: quinua, cañihua, difusividad térmica, propiedades térmicas.

## 1. EFFECT OF MOISTURE AND TEMPERATURE ABOUT THERMAL DIFFUSIVITY IN ANDEAN GRAINS


#### Abstract

This study shows a simple method for evaluating the thermal diffusivity $(\alpha)$ in Andean grains quinoa (Chenopodium quinoa Willd) and cañihua (Chenopodium pallidicaule Aellen). In addition the effect of temperature 25 to $35^{\circ} \mathrm{C}$ and humidity based humidity 10 to $20 \%$ about the behavior of this property was studied. The method used to determine the diffusivity was proposed by Gupta, which makes use of heat penetration curves in specially reinforced equipment for this purpose. The results indicate that the values vary between 7.5 and 8.9 $\mathrm{x} 10^{-8} \mathrm{~m}^{2} \mathrm{~s}^{-1}$. The evaluation of the data indicates that there is a strong influence on the type of grain, humidity and temperature in this thermal property. Additionally, the response surface methodology was used to find an empirical model to represent the thermal diffusivity depending on the moisture content and temperature.


Key words: quinoa, canihua, thermal diffusivity, thermal properties.

a. Escuela Profesional de Ingeniería Agroindustrial, Universidad Nacional de Moquegua, Av. Ancash s/n, Moquegua, Perú, b. Escuela de Postgrado Universidad Nacional Agraria la Molina, c. Universidad José Carlos Mariátegui.

e-mail: nilefox@gmail.com; Teléfono: +51-988538896

## 2. INTRODUCCIÓN

Los granos de quinua (Chenopodium quinoa Willd) y cañihua (Chenopodium pallidicaule Aellen) se cultivan por encima de los $4000 \mathrm{msnm}$, son una fuente muy rica en proteínas $(\sim 16-20 \%)$ en especial por el balance aminoácido que presenta. Por tal razón, los granos andinos forman parte de la dieta diaria de la población andina y hoy en día es demandado por el mercado internacional. Sin embargo, se puede encontrar una gran variedad de granos de quinua y cañihua dependiendo de su ecotipo, madurez biológica, localización del cultivo y composición del suelo ${ }^{1,2}$.

Los granos andinos una vez cosechados, se someten a una etapa de secado para reducir su contenido de humedad en base seca $(\sim 12 \%)$, esto permite prolongar el periodo de vida útil de los granos antes de su comercialización y consumo. No obstante los granos andinos pueden ser comercializados en su forma nativa (sin procesar) o procesados mediante diversas operaciones unitarias tales como reducción de tamaño, extrusión, tostado entre otros ${ }^{3}$. En el procesamiento de los granos andinos además de los controles tradicionales de humedad, temperatura y tiempos de proceso, la difusividad térmica, es un parámetro importante a considerar, debido a que nos permite establecer la capacidad de la transferencia de calor en estado no estacionario en operaciones como son: el secado, extrusión, expansión y tostado ${ }^{4}$. Por tal razón, su determinación permitirá incrementar la eficiencia en un proceso productivo que relacione la transferencia de calor.

La difusividad térmica, $\alpha=\mathrm{k} /(\rho . \mathrm{Cp})$, mide la tasa de difusión de calor en un material que posee conductividad térmica, $\mathrm{k}\left(\mathrm{W} \mathrm{m}^{-1}{ }^{\circ} \mathrm{C}^{-1}\right)$, densidad, $\rho\left(\mathrm{kg} \mathrm{m}^{-3}\right)$; y calor específico, $\mathrm{Cp}$ $\left(\mathrm{J} \mathrm{kg}^{-1}{ }^{\circ} \mathrm{C}^{-1}\right)$. Sin embargo, pese a su gran interés es la propiedad menos estudiada en los granos andinos, quizás debido a la escasez de equipos comerciales destinados a tal fin. No obstante, dicha propiedad térmica varía en función de la temperatura, humedad, composición proximal, densidad y porosidad 5 .

Poulsen ${ }^{6}$ y Gupta ${ }^{7}$, partiendo del método transitorio de Dickerson ${ }^{8}$, construyen las curvas de penetración de calor; la pendiente de dicha curva permite estimar el valor de la difusividad térmica en alimentos porosos a humedades bajas, considerando que la temperatura, humedad y porosidad son variables que influyen al momento de determinar la difusividad térmica en alimentos porosos. Por tales consideraciones, el objetivo del presente trabajo de investigación fue la determinación de la difusividad térmica en granos andinos de quinua variedades INIA Salcedo y Blanca de July y granos de cañihua variedades Cupi y Ramis acondicionados a niveles de humedad en base húmeda ( $\mathrm{Hbh}$ ) de 10,15 y $20 \%$ y temperaturas de 25,30 y $35^{\circ} \mathrm{C}$.

## 3. PARTE EXPERIMENTAL

Materia prima: Los granos enteros de quinua variedades INIA Salcedo y Blanca de July $(5 \mathrm{~kg})$, y granos de cañihua variedades Cupi y Ramis $(5 \mathrm{~kg})$ fueron adquiridos del almacén de semillas del Instituto Nacional de Investigación Agraria INIA, Estación Experimental
Rinconada - Salcedo - Puno. Los granos adquiridos fueron analizados en su contenido de carbohidratos, proteínas, grasas, humedad y fibra.

Los granos fueron acondicionados a contenidos de humedad de 10, 15 y 20\%. Para tal fin los granos fueron depositados en recipientes de vidrio de capacidad de $500 \mathrm{~g}$, los recipientes fueron colocados en campanas de vidrio herméticas en cuyo interior se tenía soluciones salinas que permitían controlar la humedad relativa (HR) del medio ambiente como hidróxido de sodio $(\sim 10 \% \mathrm{HR})$, cloruro de litio $(\sim 15 \% \mathrm{HR})$ y acetato de potasio $(\sim 20 \% \mathrm{HR})$. Los granos fueron pesados de forma diaria hasta alcanzar la humedad en equilibrio (peso constante).

Se determinó la gravedad especifica de los granos acondicionados mediante una balanza tipo Mohr Westphal modelo LB 502; la densidad real se calculó a partir de la gravedad especifica; la densidad aparente fue determinada por el método de desplazamiento de volumen y la porosidad fue determinada de la relación de la densidad real y densidad aparente.

Determinación de la difusividad térmica: Las figuras 1 y 2, muestran el equipo instalado especialmente para este fin según lo propuesto por Ureña 9 . El equipo consiste en un cilindro de $250 \mathrm{~mm}$ de longitud y $48 \mathrm{~mm}$ de diámetro interno, con un espesor de pared aproximado a $1 \mathrm{~mm}$. El cual está inmerso dentro de otro cilindro de mayor diámetro; en ambos extremos el cilindro tiene tapones de teflón que la sellan herméticamente, el espacio dejado por ambos cilindros es por donde circula el agua a una temperatura constante (figura 1). Uno de los tapones tiene un agujero con hilo de tornillo en el centro, por donde se introduce y se sujeta el sensor llamado Micropack, que forma parte del módulo de interface denominado DATATRACE TEMP; el cual con ayuda de un software de aplicación y un ordenador completan el sistema de adquisición de datos; la termocupla del sensor, cuyo extremo llega al centro geométrico del cilindro, y por donde se registra la temperatura de la muestra que es colocada en el interior del cilindro.



Figura 1. Esquema del cilindro Material acero inoxidable AISI 304, Cotas en mm

Los granos de quinua y cañihua acondicionados a 10, 15 y 20\% de humedad en base húmeda (Hbh) fueron introducidos dentro del cilindro (figura 1). Un sensor registra la señal de variación de temperatura en función del tiempo (precisión de $\pm 0.05^{\circ} \mathrm{C}$ ); los granos fueron evaluados a 25,30 y $35^{\circ} \mathrm{C}$. Se registró 30 datos por segundo, la figura 2 muestra el esquema del equipo usado para determinar la difusividad térmica de los granos andinos.



Figura 2. Esquema del equipo usado para determinar la difusividad térmica

Entonces, para determinar la difusividad térmica se partió de la ecuación general de transferencia de calor, que es la siguiente:

$$
\frac{\partial T}{\partial t}=\alpha\left(\frac{\partial^{2} T}{\partial x^{2}}+\frac{\partial^{2} T}{\partial y^{2}}+\frac{\partial^{2} T}{\partial z^{2}}\right)
$$

Partiendo de la ecuación 1, expresada en derivadas parciales; donde: T: es la temperatura; t: es el tiempo; $\mathrm{x}, \mathrm{y}$, z son las posiciones axiales de la transferencia de calor; se obtiene la ecuación 2 , que nos permite obtener los perfiles temperatura para una lámina y cilindro finito ${ }^{10}$. Las soluciones analíticas son soluciones de serie que contienen exponenciales, seno y funciones trascendentales. Para un cilindro finito a una temperatura inicial uniforme, expuesto a una temperatura ambiental constante y con superficie con resistencia a la convección despreciable, la solución propuesta es la siguiente ${ }^{8}$.

$$
\frac{T_{S}-T}{T_{S}-T_{i}}=\sum_{m=1}^{\infty} \sum_{n=1}^{\infty} \frac{2(-1)^{m+1}}{\beta_{m}} \cos \left(\beta_{m} 2 x / l\right) \frac{2 J_{0}\left(\beta_{n} r / R\right)}{\beta^{n} J_{1}\left(\beta_{n}\right)} \exp \left[-\left(\frac{\beta_{n}^{2}}{R^{2}}+\frac{4 \beta_{m}^{2}}{l^{2}}\right) \alpha t\right] \text { Ec. } 2
$$

Donde, $T_{S}$ : Temperatura del medio circundante, $\mathrm{T}_{\mathrm{i}}$ : Temperatura inicial, $\mathrm{x}$ : Coordenada rectangular, 1: Longitud del cilindro finito, $\mathrm{J}_{0}$ : Función bessel de primer clase de orden cero, $\mathrm{J}_{\mathrm{i}}$ : Función bessel de primer clase de orden uno, R: Radio en $(\mathrm{m}), \beta_{n}$ : Raíz de la función Bessel, $\beta_{m}$ : Raíz de la función coseno, t: Tiempo (s), r: Coordenada radial y $\alpha$ : Difusividad térmica. Para situaciones donde la muestra es expuesta por un período prolongado de tiempo, sólo el primer término de la solución por series es necesario. Para un objeto finito de forma cilíndrica, la solución expresada por la ecuación 2, puede ser simplificada de la siguiente
manera, considerando que $\mathrm{m}=\mathrm{n}=1$; es decir, $\beta \mathrm{m}=\beta / 2, \beta \mathrm{n}=2,4048, \mathrm{y}_{1}(2,4048)=0,5191$. En el centro del objeto cilíndrico, $\mathrm{x}=0, \mathrm{r}=0 ; \mathrm{y} \mathrm{J}_{\mathrm{o}}(0)=1,0$. De esta manera la solución aproximada para largos períodos de tiempo es expresada como $^{10}$ :

$$
\frac{T_{s}-T}{T_{s}-T_{i}}=2,0396 \exp \left[-\left(\frac{2,4048^{2}}{R^{2}}+\frac{\pi^{2}}{\ell^{2}}\right) \alpha t\right]
$$

Cuando se grafican las curvas de penetración de calor experimentales sobre papel semilogarítmico, es posible expresar la ecuación 3 como:

$$
t=f_{h} \log \left(j \frac{T_{s}-T_{i}}{T_{s}-T}\right)
$$

De la ecuación 4; $f_{h}=0,398 \frac{r^{2}}{\alpha}$ es el parámetro de la curva de calentamiento y $j=1,6$; para el caso de un cilindro infinito. La pendiente de la curva $\mathrm{t}=\log (\mathrm{T})$, permite determinar el valor de $f_{n}$. Reemplazando términos en la ecuación 4. Se obtiene:

$$
t=0.398 \frac{r^{2}}{\alpha} \log \left[1.6 \frac{T_{a}-T_{0}}{T_{a}-T}\right]
$$

A partir de la ecuación 5, podemos calcular la difusividad térmica como:

$$
\alpha=\frac{0.398 r^{2}}{f_{h}}
$$

De la ecuación 6; $\alpha$ es la difusividad térmica (m2 s-1), $\mathrm{r}$ es el radio del cilindro (m), fh es el parámetro de la curva de calentamiento (s-1). Los resultados de difusividad térmica para los granos fueron analizados estadísticamente empleando un arreglo multifactorial y se realizó la prueba de comparación múltiple de Tukey para observar la existencia de grupos homogéneos de datos.

## 4. RESULTADOS Y DISCUSIÓN

La tabla 1 resume el resultado de los análisis fisicoquímicos realizados a los granos de quinua y cañihua (antes de acondicionar). Se puede observar que el contenido de humedad inicial promedio fue de $\sim 8 \%$. Al respecto, diversos estudios mencionan que después del trillado y secado de los granos andinos, su contenido de humedad varía de 15 a $12 \%(3)$. Sin embargo, una vez que el grano es procesado (perlado y lavado) presenta un contenido de humedad inferior a $10 \%$, esto debido a un período de secado de 72 horas expuestos al ambiente (18 $-22^{\circ} \mathrm{C}$ ). Este descenso se debe a la ausencia del perigonio eliminado en la etapa de lavado permitiendo una mayor deshidratación ${ }^{11}$. Los valores reportados en la tabla 1 , son similares a los reportados por otros autores.
Tabla 1. Análisis fisicoquímicos de los granos de quinua y cañihua.

| Característica | Cañihua |  | Quinua |  |
| :--- | :---: | :---: | :---: | :---: |
|  | Cupi | Ramis | B. July | INIA S. |
| Humedad \% | 8,81 | $8,57\left(10,2^{*}\right)$ | 9,30 | $9,18\left(11,65^{* *}\right)$ |
| Proteína \% | 13,65 | $16,27\left(14,0^{*}\right)$ | 16,3 | $13,48\left(13,81^{* *}\right)$ |
| Grasa \% | 3,48 | $3,30\left(4,30^{*}\right)$ | 8,20 | $8,18\left(5,01^{* *}\right)$ |
| Fibra \% | 2,36 | $3,36\left(4,80^{*}\right)$ | 4,88 | $5,11\left(4,38^{* *}\right)$ |
| ELN \% | 65,07 | $60,6\left(64,0^{*}\right)$ | 56,18 | $61,53(59,74 * *)$ |

* Fuente: Abugoch12

**Fuente: Peñarrieta13

Los granos fueron acondicionados a valores de humedad promedio de 10, 15 y 20\% (tabla 2); dichos porcentajes de humedad fueron considerados porque son los rangos más empleados en los procesos de almacenamiento y transformación como son los procesos de extrusión, expandido y laminado 3,11 .

Los cálculos de densidad real y aparente permiten determinar la porosidad del lecho a diferentes niveles de humedad; esta información permite entender la forma en la cual los granos se acomodan en el lecho poroso (cilindro para evaluar difusividad térmica). Al respecto, la porosidad de los granos andinos varía de 0,23 a 0,357 (tabla 2); los valores más bajos de porosidad pertenecen a los granos de cañihua, esto por el tamaño y menor diámetro de los granos que les permite acomodarse y compactarse mejor en el lecho poroso, estas variaciones apreciadas se deben al contenido de humedad, a la forma y tamaño de los granos.

Tabla 2. Análisis de humedad y porosidad en granos.

| $\begin{array}{l}\text { Tipo de } \\ \text { grano }\end{array}$ | $\begin{array}{l}\text { Variedad del } \\ \text { grano }\end{array}$ | $\begin{array}{c}\begin{array}{c}\text { Acondicionamiento del } \\ \text { contenido de humedad } \\ \left(\% \mathrm{H}_{\mathrm{bh}}\right)\end{array} \\ \end{array}$ | Porosidad (E) |
| :---: | :---: | :---: | :---: |
| Quinua | INIA - Salcedo | 10,1 | 0,357 |
|  |  | 15,2 | 0,342 |
|  |  | 20,3 | 0,327 |
|  | Blanca de July | 10,2 | 0,339 |
|  |  | 15,3 | 0,323 |
|  |  | 20,2 | 0,314 |
| Cañihua | Cupi | 10,2 | 0,284 |
|  |  | 15,1 | 0,271 |
|  |  | 20,1 | 0,262 |
|  | Ramis | 10,3 | 0,258 |
|  |  | 15,2 | 0,246 |
|  |  | 19,9 | 0,231 |

La ASABE ${ }^{14}$ publica diferentes tipos de granos con sus respectivos porcentajes de espacios vacíos (porosidad), siendo los granos de bajo contenido de humedad los que tienen propiedades similares al grano de quinua; tal es el caso de los granos de soya (variedad Wilson), que tienen un porcentaje 0,338 de porosidad. En lo que respecta a la porosidad de los granos de cañihua su porosidad es similar a los granos de sorgo variedades Yellow Milo y Siberian con porosidades de 0,285 y 0,268 , respectivamente.

Para la calibración del equipo y validación de la metodología propuesta para evaluar la difusividad térmica (tabla 3), se determinó la difusividad térmica del agua a $20^{\circ} \mathrm{C}(\alpha$ experimental), los resultados fueron comparados con los datos de difusividad térmica del agua publicados por el Instituto Nacional de Estándares y Tecnología de EE.UU. (NIST), al que se denominó ( $\alpha$ estándar).

Tabla 3. Valores de difusividad térmica del agua a $20^{\circ} \mathrm{C}$

| Detalle | Valores promedio |
| :--- | :--- |
| $\alpha$ experimental $\left(\mathrm{m}^{2} / \mathrm{s}\right)$ | $1,5143 \mathrm{E}-02$ |
| $\alpha \alpha_{\text {estandar }}\left(\mathrm{m}^{2} / \mathrm{s}\right)$ | $1,4367 \mathrm{E}-02$ |
| $\mathrm{Fc}_{\text {Factor de corrección }}$ | 0,9588 |

La tabla 4 presenta valores de la difusividad térmica en granos de quinua y cañihua, calculada a partir de la ecuación 6, acondicionadas a diferentes contenidos de humedad (tabla 2), evaluadas a temperaturas de 25,30 y $35^{\circ} \mathrm{C}$. Los valores varían de 7,95 a $8,2 \times 10^{-8} \mathrm{~m}^{2} . \mathrm{s}^{-1}$ para los granos de quinua y de 7,25 a $8,25 \times 10^{-8} \mathrm{~m}^{2} . \mathrm{s}^{-1}$ para los granos de cañihua. La difusividad térmica en los granos andinos disminuye con el incremento del contenido de humedad (figura $3)$.

Tabla 4. Valores de difusividad térmica en granos

| $\begin{array}{c}\text { Nivel de } \\ \text { humedad } \\ \text { acondicionado }\end{array}$ | Temperatura | Difusividad térmica $\boldsymbol{\alpha} \mathbf{x} \mathbf{1 0}^{-\mathbf{8}}\left(\mathbf{m}^{\mathbf{2}} / \mathbf{s}\right)$ |  |  |  |
| :---: | :---: | :---: | :---: | :---: | :---: |
|  |  | cañihua |  | quinua |  |
|  | $25^{\circ} \mathrm{C}$ | 7,855 | 7,506 | 8,893 | 8,349 |
| $10 \%$ | $30^{\circ} \mathrm{C}$ | 8,051 | 7,920 | 8,614 | 8,524 |
|  | $35^{\circ} \mathrm{C}$ | 8,204 | 8,136 | 8,798 | 8,637 |
| $15 \%$ | $25^{\circ} \mathrm{C}$ | 7,410 | 7,536 | 8,286 | 8,204 |
|  | $30^{\circ} \mathrm{C}$ | 7,910 | 7,814 | 8,270 | 8,334 |
|  | $35^{\circ} \mathrm{C}$ | 8,177 | 8,257 | 8,526 | 8,550 |
| $20 \%$ | $25^{\circ} \mathrm{C}$ | 7,259 | 7,381 | 7,953 | 8,156 |
|  | $30^{\circ} \mathrm{C}$ | 7,773 | 7,738 | 8,008 | 8,259 |
|  | $35^{\circ} \mathrm{C}$ | 7,847 | 8,210 | 8,469 | 8,421 |

Diversos autores mencionan que el contenido de humedad y temperatura influye en la variabilidad de la difusividad térmica, tal como sucede en el caso de los granos andinos. El comportamiento anómalo de la difusividad térmica en humedades bajas es característico de los alimentos porosos, ya que presentan volúmenes de aire disperso, siendo la difusividad térmica del aire unas 150 veces mayor que la del agua líquida con valores de 2,25 x $10^{-5}$ $\mathrm{m}^{2} / \mathrm{s}$ y $1,45 \times 10^{-7} \mathrm{~m}^{2} / \mathrm{s}$, respectivamente, a $25^{\circ} \mathrm{C}$. Por lo tanto, los alimentos que tengan una alta porosidad se espera que tengan mayores valores de difusividad térmica ${ }^{15,16}$. Este comportamiento se ve reflejado en la figura 3, que muestra que a mayor porosidad mayor es la difusividad térmica en los granos andinos.



Figura 3. Variación de la difusividad térmica en función a la porosidad en granos andinos a $10 \pm 0,1^{\circ} \mathrm{C}$.

La figura 4 muestra la influencia de la humedad sobre la difusividad térmica, existiendo una reducción de esta propiedad conforme se incrementa el contenido de agua en los granos andinos. Al respecto, Kostaropoulos y Saravacos ${ }^{15}$ afirman que la reducción de la difusividad térmica en la región II (humedad 5-30\%), es la región del diagrama de cambios generalizados de las propiedades de transporte en función de la humedad en alimentos porosos; dicha humedad es adsorbida en capas multimoleculares y los capilares se llenan gradualmente con agua, desplazando el aire, la difusividad térmica es reducida, subsecuentemente la difusividad térmica del agua líquida es más baja que la del aire.

Estos comportamientos anómalos de la difusividad térmica en función de la humedad y porosidad fueron advertidos por diversos estudios, los cuales mencionan que son escasos los datos en alimentos secos y semi-secos, en humedades inferiores al 30\%. Por ejemplo, un estudio ${ }^{16}$ encontró que la difusividad térmica en granos de maíz se incrementa conforme se incrementan la temperatura alcanzando valores de $0,91 \times 10^{-7}$ a $3,23 \times 10^{-7} \mathrm{~m} 2 . \mathrm{s}^{-1}$. No obstante otro estudio realizado en granos de soya encontró que conforme se incrementa el
contenido de humedad (14 a 30\%) disminuye la difusividad térmica, efecto contrario sucede con la temperatura $(50 \text { a } 150)^{17}$. Esto nos permite concluir que la difusividad térmica es un parámetro que además de ser influenciado por la porosidad, humedad y temperatura también depende de la microestructura, composición química y características físicas del alimento.



Figura 4. Variación de la difusividad térmica en función humedad en granos andinos

$$
\text { a } 10 \pm 0,1^{\circ} \mathrm{C} \text {. }
$$

Los resultados de la difusividad térmica de granos de quinua y cañihua (tabla 4) fueron analizados mediante la metodología de superficie de respuesta (figuras 5 y 6); con la finalidad de poder aproximar un modelo matemático (ecuaciones 7 y 8), que permita predecir dicha propiedad a intervalos de humedad $(10$ a $15 \%)$ y temperaturas de $\left(25\right.$ a $\left.35^{\circ} \mathrm{C}\right)$.



Figura 5. Superficie de respuesta del efecto de la humedad y la temperatura sobre la difusividad térmica de la quinua, a través del modelamiento cuadrático

$$
\begin{aligned}
\alpha_{\text {quinua }}= & 1.0334 \times 10^{-7}-7.04 \times 10^{-10} \times H-1.1635 \times 10^{-9} \times \mathrm{T} \\
& +7.854 \times 10^{-12} \times H^{2}+4.2626 \times 10^{-12} \times H \times T+2.4059 \times 10^{-11} \times T^{2}
\end{aligned}
$$



Figura 6. Superficie de respuesta del efecto de la humedad y la temperatura sobre la difusividad térmica de la cañihua, a través del modelamiento cuadrático

$$
\begin{aligned}
\alpha_{\text {cañihua }}= & 5.1431 \times 10^{-8}-5.7307 \times 10^{-10} \times H+1.5898 \times 10^{-9} \times \mathrm{T} \\
& -1.0936 \times 10^{-11} \times H^{2}+2.191 \times 10^{-11} \times H \times T-2.1187 \times 10^{-11} \times T^{2}
\end{aligned}
$$

Ec. 8

Dónde: $\alpha=$ Difusividad térmica (m2s-1); $\mathrm{H}=$ Humedad (10 - 20\%); $\mathrm{T}=$ Temperatura (25$35^{\circ} \mathrm{C}$ ), las ecuaciones 7 y 8 , desarrolladas mediante la función cuadrática (superficie de respuesta de segundo orden), nos permiten realizar interpolaciones en un rango de 25 a 35 ${ }^{\circ} \mathrm{C}$ de temperatura y de 10 a $15 \%$ de humedad; se observa en las figuras 4 y 5 que conforme se incrementa la temperatura de 25 a $35^{\circ} \mathrm{C}$ el valor de la difusividad térmica es mayor; sin embargo a niveles de 10 a $20 \%$ de humedad su valor se reduce.

El análisis de varianza, a un 95\% de confiabilidad, reporta que existe variación estadística significativa entre los tipos de granos. No obstante, las demás fuentes de variación (humedad y temperatura) presentan también diferencia estadística significativa, demostrando la dependencia que existe de la difusividad térmica entre la humedad y temperatura. El análisis de la Prueba Tukey reporta que los promedios de la difusividad térmica de los granos de quinua, variedades INIA-Salcedo y Blanca de July (quinua), son diferentes a los granos de cañihua variedades Cupi y Ramis (cañihua). Por lo tanto, se puede generalizar y concluir que la difusividad térmica varía en los granos de quinua y cañihua en función de su temperatura y humedad.

## 5. CONCLUSIONES

Se comprobó que los valores de difusividad térmica de los granos de quinua y cañihua están comprendidos entre 7,95 y $8,79 \times 10-8 \mathrm{~m}^{2} . \mathrm{s}^{-1}$ y 7,25 a $8,20 \times 10-8 \mathrm{~m}^{2} . \mathrm{s}^{-1}$; con porosidades de 0,33 a 0,35 y 0,25 a 0,28 , respectivamente; esta propiedad presenta diferencias entre los granos de quinua y cañihua por la forma y tamaño de los mismos; comprobándose la relación directa con la temperatura e inversa con el contenido de humedad en rangos de 20 a $35^{\circ} \mathrm{C} \mathrm{y}$ de 10 a $20 \%$ de humedad.

## 6. AGRADECIMIENTO

Agradecimiento a la Escuela de Posgrado y a la Facultad de Industrias Alimentarias de la Universidad Nacional Agraria La Molina, por facilitarnos los laboratorios y equipos para el trabajo de investigación, igualmente al Instituto Nacional de Innovación Agraria - INIA por las semillas de granos andinos.

## 7. REFERENCIAS BIBLIOGRÁFICAS

1. Repo-Carrasco-Valencia RA-M, Serna LA. Quinoa (Chenopodium quinoa, Willd.) as a source of dietary fiber and other functional components. Ciência e Tecnol Aliment. 2011;31(1):225-30.
2. Domínguez SS. Quinoa-Postharvest and Commercialization. Food Rev Int. 2003;19(3):191-201.
3. López LM, Capparelli A, Nielsen AE. Traditional post-harvest processing to make quinoa grains (Chenopodium quinoa var. quinoa) apt for consumption in Northern Lipez (Potosí, Bolivia): Ethnoarchaeological and archaeobotanical analyses. Archaeol Anthropol Sci. 2011;3(1):49-70.
4. Nesvadba P, Houška M, Wolf W, Gekas V, Jarvis D, Sadd PA. Database of physical properties of agro-food materials. J Food Eng. 2004; 62(1) 497-503.
5. Poulsen KP. Thermal diffusivity of foods measured by simple equipment. J Food Eng. 1982;1(2):115-22.
6. Gupta TR. Thermal diffusivity measurements of wheat flour and wheat flour dough. Food Process Eng. 1996;19:343-52.
7. Dickerson WR. An Aparatus for the Measurement of Thermal Diffusivity of foods. Food Technol. 1965;18(5):343-53.
8. Ureña P. M. Obtención de alimentos modelo a partir de mezclas bentonita: glicerol: agua para estudios de tratamientos térmicos [Tesis Doctoral]. Valencia: Politecnica e Valencia; 1990.
9. Singh RP. Thermal diffusivity in food processing. Food Technol. 1982;36(2):97-91.
10. Jancurová M, Minarovičová L, Dandár A. Quinoa - A review. Czech J Food Sci. 2009; 27(1): 71-9.
11. Abugoch James LE. Quinoa (Chenopodium quinoa Willd.): Composition, chemistry,
nutritional, and functional properties. Adv Food Nutr Res. 2009; 58:1-31.
12. Peñarrieta JM, Alvarado JA, Åkesson B, Bergenståhl B. Total antioxidant capacity and content of flavonoids and other phenolic compounds in canihua (Chenopodium pallidicaule): An Andean pseudocereal. Mol Nutr Food Res. 2008;52(6):708-17.
13. ASABE. Properties T. Products G. ASAE: Thermal Properties of Grain and Grain Products; 2008.
14. Kostaropoulos AE, Saravacos GD. Thermal diffusivity of granular and porous foods at low moisture content. J Food Eng. 1997;33(1-2):101-9.
15. Dotto GL, Pinto LAA, Moreira MFP. Determination of the effective thermal diffusivity in a porous bed containing rice grains: effects of moisture content and temperature. Heat Mass Transf. 2015; 52(4):887-96.
16. Azadbakht M, Khoshtaghaza MH, Gobadian B. Thermal Properties properties of soybean pod as a function of moisture. Am J Food Sci Technol. 2012;8(4):1217-28.
